\title{
Lectura y escritura literarias en la Universidad: desafíos y propuesta para la articulación de un nuevo espacio para el desarrollo de la práctica literaria en la enseñanza superior universitaria
}

\author{
Fabián Gabriel Mossello, Universidad Nacional de Villa María, Argentina
}

\begin{abstract}
Resumen: Las prácticas de la lectura y la escritura han sufrido, en los últimos años, profundas transformaciones en respuesta al mundo post-industrial cambiante que nos enfrentamos como maestros. La ampliación del objeto literario a nuevos espacios de expresión cultural, está revisando conceptos como el canon, la belleza, el género, el papel del lector, así como la reformulación de lo que significa para leer y escribir literatura en un contexto universitario. En este sentido, consideramos que si la universidad se ha adaptado a estos diversos escenarios culturales e históricos con el fin de ser capaz de formar lectores y escritores eficaces en el nuevo contexto cultural y más específicamente en el literario. Probablemente deberíamos revisar nuestro papel mediador en el aula para que podamos articular productivamente las expectativas de lectura y escritura a cada estudiante tiene y la oferta académica de la universidad. En este artículo vamos a tratar con los principales problemas relacionados con la lectura y la escritura de la literatura en la Universidad; y vamos a elaborar una propuesta para un taller curricular articulando estas dos actividades, mientras que la integración de las contribuciones específicas didácticas en la enseñanza de la literatura y disciplinas como la teoría literaria, la semiótica y la crítica.
\end{abstract}

Palabras clave: universidad, taller, lectura, escritura, literatura

\begin{abstract}
The practices of reading and writing have suffered, in recent years, profound transformations in response to the changing post-industrial world we face as teachers. The expansion of the literary object to new spaces of cultural expression, is reviewing concepts such as canon, beauty, genre, the role of the reader, as well as the reformulation of what it means to read and write literature in a university context. Regarding this, we consider whether the university has adapted itself to these varied cultural and historical scenarios in order to be able to form effective readers and writers in the new cultural context and more specifically in the literary one. We should probably review our mediating role in the classroom so we can productively articulate the reading and writing expectations every student has and the academic offer of the university. In this paper we will deal with the main problems concerning the reading and writing of literature at the University; and we will draft a proposal for a curricular workshop articulating these two activities, while integrating the specific didactic contributions on the teaching of literature, and disciplines such as literary theory, semiotics and criticism.
\end{abstract}

Keywords: University, Workshop, Reading, Writing, Literature

"La literatura es el mejor camino para humanizar al hombre"

Iber Verdugo

\section{Introducción}

$\mathrm{L}$ a tarde se dibujaba sobre las serranías cordobesas y el campus universitario. La literatura era todavía un mapa inescrutado; la mayoría sólo había leído lo necesario para salvar una vocación avizorada. Pero allí estábamos, un poco más de cien ingresantes a la carrera de

Revista Internacional de Ciencias Humanas

Volumen 4, Número 2, <http://lascienciashumanas.com>, ISSN 2530-4526

(C) Global Knowledge Academics. Fabián Gabriel Mosello.

Todos los derechos reservados. Permisos: soporte@gkacademics.com

Recuperado de Revista Internacional de Humanidades 4(2), 2015 (pp. 255-261) 
Letras Modernas en una universidad del centro de Argentina. Cabezas en alta frecuencia, como dijo Vicente Huidobro, manos aleteantes, como lo hubiera dicho Miguel Hernández, corazones silbando en los bordes de las letras. Y entonces, un profesor nos empezó a hablar de literatura. Era un hombre mayor, el más renombrado de los profesores de esa facultad. Y nos dijo muchas cosas que he olvidado en parte, otras sólo se han grabado en las retinas del recuerdo, pero una sigue estando después de tres décadas de enseñanza de, justamente, la literatura: "la literatura es el mejor camino para humanizar al hombre". Y esas palabras vienen rodando desde entonces y marcando cada tramo de las cosas que he venido haciendo desde aquella soleada tarde de un brumoso 1985.

Este trabajo intenta una reflexión, en parte teórica, en parte práctica, sobre la enseñanza de la literatura en el Nivel Superior a través de un nuevo espacio que propone, no sólo el desarrollo de un conocimiento a fijar y replicar, sino la compleja trama de la educación humanística, es decir, el paso necesario a la enseñanza del arte de la palabra que permite el desarrollo de la sensibilidad, el criterio estético y la reflexión sobre el hombre, la vida, el mundo que toda buena literatura no deja de referir.

\section{La pregunta}

Las nuevas consideraciones sobre el lenguaje, en particular desde la segunda mitad del siglo pasado, en el marco también del desarrollo de disciplinas como la pragmática, la sociolingüística, la teoría de la enunciación, entre otras, han abierto nuevos horizontes para trabajar lo literario en los distintos niveles de la educación. Si nos centramos en la enseñanza de la lectura y la escritura de literatura, ésta ha sufrido una serie de cambios sustanciales que permitieron salir de las viejas recetas de la imitación y/o reproducción, para instalar la idea de que leer y escribir suponen procesos interactivos y transactivos que involucran al sujeto durante todo el proceso de aprendizaje, como afirma Kenneth S. Goodman (1986).

De todos modos, y a pesar de que han pasado muchos años y muchas reformas educativas, en el contexto de la Educación Superior Universitaria no son frecuentes los espacios para la lectura de literatura ajustados a los cambios que propone este nuevo paradigma.

Podemos, sin incurrir en generalizaciones, considerar dos tipos de espacios que coexisten dentro de las universidades y que tienen como actor necesario las carreras especializadas, es decir, los Profesorados y Licenciaturas en Lengua y Literatura. Dos propuestas diferenciadas por sus objetivos, metodologías y modos de evaluar.

Por un lado, dominan el escenario los clásicos espacios para la enseñanza de la literatura sujetos a la lógica de la cátedra universitaria; espacios diferenciados por sus temas y corpus -literatura argentina, latinoamericana, europea, norteamericana, entre otras- pero coincidentes en sus metodologías. En general, salvo algunas excepciones, estos espacios están marcados por enfoques conductistas de la enseñanza, por la evaluación sumativa, y la reproducción de conceptos. En algunos casos se enfatiza algún tipo de crítica literaria particular -comparatista, estilística, política o semiótica- aunque es decisivo el enfoque histórico.

Por otro, y como respuesta a aquella enseñanza centrada en el contenido, y en sintonía con aquellos primeros experimentos educativos de la Escuela Nueva ${ }^{1}$, entre otros, aparecen las propuestas pedagógicas de los llamados espacios extracurriculares para la creación literaria. Designados de múltiples maneras, pero que recuerdan a muchos proyectos no necesariamente educativos, aunque con historia -café literario, salón literario, tertulia literaria- se fueron permeando en los ámbitos universitarios, casi siempre como proyectos de extensión. Estos espacios construidos a partir de la idea romántica del lector libre, permiten al estudiante poder decir "me parece", "a lo mejor", "esto puede ser". Así, del rigor conceptual de la cátedra de literatura hemos saltado a la libertad sin fronteras de estos nuevos espacios del placer.

\footnotetext{
${ }^{1}$ La Escuela Nueva es un movimiento pedagógico surgido a finales del siglo XIX y que proponía una educación sujeta a los principios de Jean- Jacques Rousseau, es decir, una educación abierta a lo natural, la espontaneidad, la intuición y demás principios defendidos por el romanticismo.
} 
De alguna manera, la Universidad resuelve la falta de ámbitos para la creación y la lectura literarias con estas propuestas extracurriculares, sujetos a la idea del acercamiento a la literatura como actividad sensibilizadora o lectura por placer y que supone, en casi todos los casos, o leer producción personal de los mismos alumnos (no los podemos llamar talleristas pues no son talleres), o de autores clásicos de la literatura universal (latinoamericana, norteamericana, europea, entre otras).

Sin embargo, entre estas dos opciones falta una tercera: el del taller como espacio germinal del conocimiento; un espacio en el que leer y escribir son considerados trabajos (Barthes, R, 1981: 44); un trabajo artesanal que involucra proceso cognitivos complejos. Leer literatura es una práctica social que compromete al tallerista en múltiples dimensiones -cognitiva, pragmática, patémica-. En este caso,

la modalidad de taller, [aporta] desde la práctica más que desde la teoría la concepción de texto móvil, nunca cerrado definitivamente, siempre en estado de versión modificable. Cada lectura de un texto producido en taller revela a su autor las múltiples lecturas posibles y lo invita a revisar, modificar y mejorar hasta donde decida" (Finocchio, 2009: 6).

Así, sobre la base de una lectura que resignifica el objeto, es decir, una lectura multiplicadora, la práctica de taller potencia el desarrollo de estrategias y habilidades que van más allá de la mera reproducción de contenidos.

\section{Presupuestos para una propuesta de taller en la Universidad}

La lectura y la escritura de literatura pueden ser abordadas teniendo en cuenta estas ideas en un entorno específico del aula universitaria. Espacio que supone, por lo general, un grupo heterogéneo de alumnos (estudiantes de Música, Cine, Sociología, Ciencias Políticas, idiomas o Letras, entre otras carreras), situación que dinamiza la propuesta y diversifica las motivaciones.

En primer lugar debemos partir, como ya se dijo, de la idea de que la lectura y la escritura son trabajos (Barthes, 1981: 32) que implican estrategias del sujeto frente al lenguaje. Leer no es sólo decodificar, y escribir, usar una tecnología. Son prácticas densas, complejas que involucran al sujeto en distintos órdenes de su praxis. Esto supone una segunda idea: considerar la lectura y la escritura como trabajos del pensamiento, de la acción, de la pasión. Una propuesta 'volumétrica', en la que los tres órdenes de la práctica se imbrican.

En tercer lugar, estos órdenes no deben interacturar sólo para resolver una consigna -en tanto producto-. Es necesario partir de la idea de que la práctica de escritura es también un proceso que debe propiciar reflexiones sobre el mismo hacer. Siguiendo el "modelo propuesto por Flower y Hayes, la composición de un escrito se realiza a partir de tres operaciones básicas (planificación, textualización y revisión) recursivas, pues no suponen una secuencia sino que varían de acuerdo con la situación de escritura que plantea cada texto en particular" (Finocchio, 2009: 9).

En cuarto lugar, leer y escribir en la Universidad deben ser prácticas intertextuales, interdiscursivas e interdisciplinarias. Tanto la resolución del problema que supone la lectura de literatura, como la concreción de un proyecto de escritura deben abrirse a otras perspectivas que retomen los problemas de género, de los límites entre ficción y no ficción, del lector, del lenguaje, de las conexiones con el campo literario, cultural e histórico, entre otros. Un diálogo abierto con saberes disciplinares como los de la teoría literaria, las historias de la literatura, la crítica, las didácticas específicas. Esto permite complejizar las prácticas, densificar sus alcances y redimensionarlas significativamente en contextos de la Educación Superior.

En quinto lugar, la reflexión sobre la escritura y la lectura permite superar 'el momento sensibilizador' para propiciar la construcción de conocimiento. Pero, ¿qué ocurre cuando resolvemos una consigna de lectura o de escritura?, ¿el sujeto es el mismo después del desafío que suponen estas prácticas? Nuestro punto de partida es la afirmación de que leer y escribir transforman al sujeto, modifican su visión de mundo, y aportan nuevas formas de conocer.

En sexto lugar, afirmamos, que lejos de la idea de que la literatura es un tipo de discurso excluyente por la naturaleza de su lenguaje, los temas, y el espacio de circulación, la propuesta que 
realizamos supone considerar la lectura y la escritura como prácticas inclusivas, atravesadas por los itinerarios individuales y colectivos de una cierta comunidad. Esto implica pensarlas como haceres sometidos a las tensiones que articulan, no sólo el campo literario, sino también social, histórico e ideológico que influyen en cada individuo. Todos pueden escribir, todos pueden leer; basta sólo proponerse el ejercicio artesanal (Barthes) de hilvanar palabras, de ejecutar la partitura del lenguaje, de saborear las páginas "levantando la cabeza" (Barthes, 1981: 42) para buscar las reminiscencias del texto. Esto abre un séptimo punto en relación al delicado asunto del canon, pues ¿qué leemos para sostener la propuesta?

Martín Kohan afirma al respecto que

la temática del canon literario toca un aspecto fundamental del trabajo de los docentes (pero también del trabajo de los escritores, los críticos, los periodistas culturales, los bibliotecarios, los editores, los traductores, los sociólogos, etc.), en la definición del canon se dirimen centralidades y periferias, valores y disvalores literarios, consagraciones y postergaciones, pedestales y olvidos; también se determina qué literatura va a ser leída y qué literatura no va a ser leída, y de qué manera va a ser leída la que sea leída (dentro de qué tradición, con qué categorías, con qué sentidos); en la definición del canon literario se dirime también una manera de concebir una identidad (aquella a la que una determinada literatura puede interpelar; ya sea por ejemplo la literatura latinoamericana, o la literatura judía, o la literatura argentina, o la literatura universal, etc.)" (Kohan Martín, 2009: 8).

Al respecto Ana María Finocchio reflexiona que "la biblioteca no es fija, (...). Cada mudanza siempre trae un nuevo ordenamiento, una recolección" (Finocchio, 2009: 11). Estas ideas del canon como espacio móvil deben iluminar nuestra práctica de taller que no se podrá restringir únicamente al campo de lecturas y las propuestas de escritura en base a autores clásicos (para nuestro campo literario -Jorge Luis Borges, Marechal, Roberto Arlt, Puig-). Es necesario abrir el diálogo a autores que invoque otras estéticas, soportes, y circuitos de lectura, digamos en Argentina: Haroldo Conti, Daniel Moyano, Roberto Fontanarrosa, entre otros, multiplicando los estilos y los géneros historieta, cine, teatro-. La dinámica del campo de lecturas es una de las claves de este tipo de taller; un canon abierto y permeable permite el juego de las lecturas posibles, enriquece las categorías problemáticas y ofrece al tallerista una visión más amplia de qué es la literatura.

Un punto importante en estos talleres universitarios es el paso necesario de lo discursivo a lo social, cultural, e histórico, que supone considerar al texto, además de un lugar donde construir lo literario, un momento para contar sobre lo que le pasa al tallerista. En estos talleres de literatura es frecuente el salto a las historias de vida, rebasadas por los datos que provienen del contexto crítico de producción. Emergen, entonces, textos que constituyen, como dice Leonor Arfuch (2010) "las narrativas del yo, que configuran un espacio biográfico no como una mera sumatoria de géneros diversos, "nobles o plebeyos", sino como un horizonte de inteligibilidad para leer, sintomáticamente, tendencias de la cultura y de las formas de ser y hacer de nuestro tiempo" (Arfuch, L., 2009: 6). En definitiva, la lectura y la escritura desembocan inevitablemente en el sujeto, es decir, en un recorrido que retorna al Yo. Por todo, consideramos que este modo de leer y escribir literatura propende a la construcción de subjetividades que en su sutura (Hall, Stuard, 2003: 63) modela las identidades.

Por último, el taller que describimos es curricular. Es decir, integra el conjunto de asignatura que debe aprobar un alumno regular. Por lo tanto se debe evaluar, colocar una o varias notas. Nos preguntamos: ¿cómo evaluar la creatividad, la imaginación? Este aspecto lo desarrollaremos en el apartado siguiente.

Los lineamientos mostrados no constituyen niveles a cumplir de manera secuenciada. Más bien tienen la intención de proponer una paleta de aspectos a tener en cuenta, como líneas de acción para organizar unas prácticas -leer y escribir- en relación con un objeto a priori problemático -la literatura- en un contexto tan rico como complejo -la Educación Superior Universitaria-. Como dice Maria Pilar Gaspar, 
Una intuición que suele circular socialmente y que ha tenido fuerte impronta en las prácticas [educativas] (...) es la de que la lectura constituye un acto por medio del cual se "decodifica", esto es, a partir de los signos gráficos se asocian esos signos con sonidos y con significados. De la misma manera, se suele considerar que escribir es "volcar las ideas sobre el papel" (...). Lo cierto es que leer es mucho más que decodificar y escribir no es sólo trazar signos que representan ideas. Por tanto, leer y escribir (y también hablar y escuchar) son tareas que ponen en juego muchos otros conocimientos. Es función de [las instituciones educativas] (...) (y de todos los docentes) colaborar para que los alumnos aumenten los conocimientos involucrados en las tareas de lectura y escritura". (Gaspar, M.P, 2009: 6)

¿Pero, cómo articular estas reflexiones con la práctica concreta de la lectura y la escritura creativa en la Universidad? ¿Qué particularidades tiene este espacio que debemos atender?

\section{Reflexiones para una buena práctica de taller}

El espacio del aula universitaria supone, por lo general, un grupo heterogéneo de alumnos (estudiantes de Música, Cine, Sociología, Ciencias Políticas, idiomas o Letras, entre otras carreras), situación que dinamiza la propuesta y diversifica las motivaciones.

De este modo y como consecuencia de estos cruces y divergencias, la literatura en los estudios Superiores Universitarios discurre entre distintos campos disciplinares: la historia de la literatura; la crítica y la teoría literaria; las didácticas específicas, entre otras asignaturas. El taller que proponemos debe establecer un diálogo con estas disciplinas, en una apertura a sus metodologías, sus objetos específicos, además de la literatura, sin que esto suponga interferencia de contenidos y actividades. De la teoría literaria se retomarán sus preguntas: ¿qué es la literatura?, ¿qué es y cómo se constituye en canon literario? De la crítica, las formas de 'acceso al texto'. De la historia literaria, los recortes de corpus, las generaciones, movimientos, grupos y escuelas.

Pero también leer es también elegir qué leer. La constitución del campo de lecturas es un asunto clave en la dinámica del taller universitario. Sobresalen las preguntas y los criterios: ¿históricos?, ¿por autores?, ¿estéticos? La misma constitución heterogénea del espacio universitario modela el canon. Lejos de ejes fijos o más estructurados, como aparece en otras asignaturas, el coordinador de un taller de lectura y escritura puede modelar activamente su campo de lecturas en relación a intereses y respuestas del tallerista a los temas y géneros propuestos. Esta libertad permitirá hacer circular los objetos literarios según la apreciación del lector y así conformar recorridos personales de lecturas.

Desde la perspectiva de los aprendizajes, leer implica un modo de preguntar. En un taller de lectura y escritura literarias universitario valen todas las preguntas posibles. Partimos de la idea de que las prácticas de lectura y escritura deben propiciar procesos, tanto cognitivos como pragmáticos y patémicos. Leer debe ser una instancia abierta al diálogo, 'levantando la cabeza' como sugería Barthes (1981). Dada la heterogeneidad esencial del alumnado de la universidad, el tipo de devolución es multidireccional y multiplicadora. En un mismo curso conviven saberes, edades, actitudes y aptitudes disímiles, por lo que la lectura de literatura en la universidad es un desafío para el que coordina, pero también fuente de recorridos muchas veces reveladores.

Cuando en las distintas áreas curriculares se aborda la lectura de un texto, cabría preguntarse para cada caso particular, como afirman algunos especialistas: ¿qué queremos que los alumnos comprendan de este texto?, ¿es importante la afectividad en la comprensión? Estas preguntas tienen derivaciones metodológicas y suponen una convicción. Tal vez no habría que pretender que la lectura que realicen los alumnos agote todo lo que el profesor-coordinador comprende al leer el mismo texto. Tal vez, a veces podamos orientar la lectura sólo para que presten atención a un aspecto o concepto (tal como hacemos todos los lectores al leer algunos textos, por ejemplo, en busca de alguna información específica). En ese caso, no es necesario hacer un trabajo exhaustivo, sino simplemente colaborar para que logren "la conceptualización que, desde la enseñanza, se ha priorizado" (Gaspar, M. P, 2009: 8).

De todos modos, es necesario no olvidar un asunto central: la evaluación. Como referimos en el apartado anterior, el taller que describimos es una asignatura del plan de la carrera, por lo que el estudiante deberá cursar y regularizar a través de distintas instancias evaluativas. Creo que en 
términos todavía provisionales, se puede calificar en un taller de lectura y escritura creativas atendiendo a distintos aspectos de la práctica:

* Al proceso de lectura y escritura. Se puede evaluar la planificación, el recorrido y los resultados de ese proceso, con aciertos y errores. El error es parte del proceso, por lo que es importante evitar las calificaciones, al menos en un principio, del tipo sumativas.

* Al producto. Si empezamos diciendo que la escritura (y también la lectura) se ajustan a un plan cognitivo que implica pruebas, ensayos, marchas y retrocesos, es de esperar que en algún punto del trabajo se concrete un producto (provisional) pero producto al fin, evaluable. Evaluar textos en este nivel puede ser una oportunidad, no sólo para considerar una nota, sino también revisar detalles y pulir aspectos para futuras prácticas. Así, "escribir siempre es reescribir, por lo tanto, es necesario convertir nuestra práctica de corrección en práctica de lectura o revisión de los textos de nuestros alumnos para orientar su reescritura" (Finocchio, A.M., 2009: 11).

En estos talleres se debe trabajar con los borradores, propiciando la lectura en clase, la discusión de cada texto, a fin de mostrar que la literatura es siempre un camino por recorrer y no sólo una meta a la que llegar.

Por último, escribir es un hacer multiplicador para las instancias del decir. En relación con la escritura sucede algo parecido que con la lectura. Los talleres universitarios son espacios de alta permeabilidad de temas, géneros y estéticas, para citar algunos aspectos. Un estudiante de una Licenciatura en Música es probable que apele a recursos diferentes a los que podría apelar uno de Letras o Cine. En este sentido, la escritura de

la literatura pone en juego una serie de saberes y conocimientos propios y particulares de cada época. Expone, con ese particular modo de entramar las palabras, una serie de temas y los hace trabajar. El amor, la muerte, la soledad, la envidia, el narcisismo, el holocausto, la hermandad (...). La literatura tiene esa virtud de hablar de todos esos temas; incluso cuando no existe otro modo de referirse a esos temas, la literatura siempre puede hacerlo. Cuando la censura se aplica, sólo el discurso literario es capaz de continuar hablando de lo que está prohibido. Por eso la literatura es peligrosa. Por eso hay que vigilarla, cercar unos contenidos que se consideren apropiados y callar otros (Cano, Fernanda, 2010: 11).

\section{Conclusión}

Enseñar la lectura y la escritura de literatura en la universidad es un desafío todavía abierto que supone una multitud de caminos por recorrer. Desafío para los profesores que deben estimular su inteligencia para entender el cambiante mundo de las aulas universitarias; desafío para el alumno que se encontrará con un espacio de consignas a resolver que muchas veces ponen en juego su propia cultura.

Así, la literatura, ese discurso que calla, afirma, discute, refuta y reescribe cosmovisiones, es un buen punto de partida para humanizar la enseñanza en la universidad a través de estos talleres que seguimos pensando. 


\section{REFERENCIAS}

Alvarado, M. (coordinadora) (2001). Entre Líneas. Teorías y enfoques en la enseñanza de la escritura, la gramática y la literatura. Buenos Aires: FLACSO-Manantial.

Arfuch, L. (2009). Historias de vida: subjetividad, memoria y narración. Buenos Aires: FLACSO.

Barthes, R. (1984). Los susurros del lenguaje. BsAs: Edicial.

Cano, F. (2010). Sobre la enseñanza de la literatura (o de las tensiones entre el-quehacer docente y el quehacer lector). Buenos Aires: FLACSO.

Eco, U. (1965). Obra Abierta. Buenos Aires: Seix Barral.

Flower, L. y Hayes J. (2011). Textos en contexto. Los procesos de lectura y escritura. Buenos Aires: FLACSO.

Gaspar, M. P. (2009). La lectura y la escritura en el proyecto escolar (o de cómo la lectura y la escritura no son patrimonio de un área). Buenos Aires: FLACSO.

Goodman, K. (1986). La lectura, la escritura y los textos escritos. Universidad de Arizona. Mímeo.

Hall, S. (2003). Cuestiones de identidad cultural. Buenos Aires: Amorrortus.

Kohan, M. (2009). Notas sobre el canon. Buenos Aires: FLACSO.

Mossello, F. y Melana, M. (2007). Construcción de identidades culturales a partir de procesos de escritura de ficción. Córdoba, Argentina: Advocatus.

Rulfo, J. (1953). Nos han dado la tierra. México: FCE

Sarlo, B. (compiladora) (1981). El mundo de Roland Barthes. BsAs: Cátedra.

\section{SOBRE EL AUTOR}

Fabián Gabriel Mossello: Profesor de Teoría, Semiótica y Crítica Literaria en la Universidad Nacional de Villa María, Córdoba, Argentina. Coordina los talleres de lectura y escritura creativa del Módulo de Arte I y II, Sede Córdoba de la UNVM. Ha estudiado en la Universidad Nacional de Córdoba, en la que se graduó como Profesor y Licenciado en Letras Moderna. Más tarde como Magíster en Literaturas Latinoamericanas y después como Especialista en Ciencias Sociales, con mención en Lectura, Escritura y Educación, FLACSO (Bs.As.). Entre sus libros se destacan: Literatura, Ideología y Sociedad. Argentina: EDUVIM, 2010; Imaginarios literarios y culturales (en coatoría). Argentina: El Copista, 2011. Lugares del decir (en coatoría). Argentina: Homo Sapiens. Se está doctorando en semiótica por el CEA, Cba. Con el tema el Neopolicial en Argentina. 